\section{Commentary: Dissecting surgical systems}

\author{
Todd L. Demmy, MD
}

\begin{abstract}
Rajaram and colleagues ${ }^{1}$ have worked hard to balance numerous relevant covariates in the Premiere database to show an expected reduced pain medication use for less invasive approaches and a putative advantage for robotic lobectomy over VATS of less magnitude. This contribution is difficult to translate into clinical practice because approach is only one element of complex systems designed to reduce pain and complications. A sampling of these elements is depicted in Figure 1. Many elements are applied differently, adapting to idiosyncrasies in surgeon training, preferences, and resources.
\end{abstract}

Surgical "systems" adapting tools to the complexities of pathology were described before the 19th century and now these intersect with other expansive fields like anesthesia and pain management. ${ }^{2,3}$ Surgeons and hospital systems will tend to do better when targeting numerous pain mechanisms rather than focusing on one element, such as approach. This is akin to addressing many technical and perioperative details to have a successful esophageal or transplantation program. Systems often have scripted responses to common complications that limit their deleterious effects.

Some thoracotomy patients have minimal pain, whereas occasional video-assisted thoracoscopic surgery (VATS)/ robotic neuralgias are severe. Although multifactorial and difficult to predict like an esophageal conduit leak, severe pain factors within surgeons' control are not systematically captured. Both VATS and robotic classifications encompass a diverse universe of incision sets and instruments, causing different levels of pain stimulation depending on nerve proximity. ${ }^{4,5}$ Then minimally invasive

\footnotetext{
From the Department of Thoracic Surgery, Roswell Park Cancer Institute, Buffalo,

NY; and Department of Surgery, University at Buffalo, Buffalo, NY.

Disclosures: Dr Demmy serves as a consultant for Medtronic.

The Journal policy requires editors and reviewers to disclose conflicts of interest and to decline handling or reviewing manuscripts for which they may have a conflict of interest. The editors and reviewers of this article have no conflicts of interest.

Received for publication June 4, 2020; revisions received June 4, 2020; accepted for publication June 5, 2020; available ahead of print June 27, 2020.

Address for reprints: Todd L. Demmy, MD, Department of Thoracic Surgery, Roswell Park Cancer Institute, Elm and Carlton St, Buffalo, NY 14263 (E-mail: todd. demmy@ roswellpark.org).

J Thorac Cardiovasc Surg 2021;162:271-2

0022-5223/ $\$ 36.00$

Copyright (C) 2020 by The American Association for Thoracic Surgery

https://doi.org/10.1016/j.jtcvs.2020.06.053
}

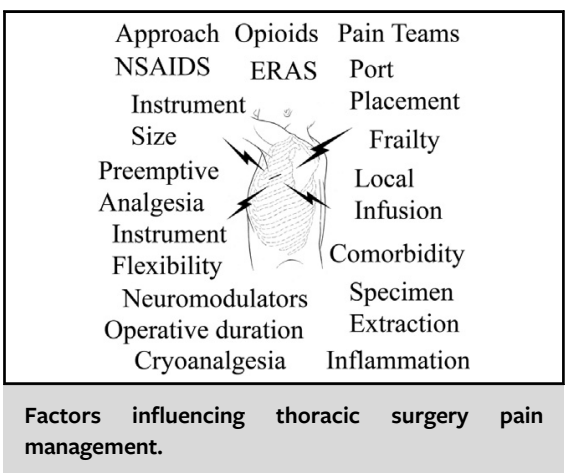

CENTRAL MESSAGE

Administrative databases need to

capture many more pain-related

variables that evolving diverse

surgical systems target before

statistical analyses can

adequately relate key elements

like approach.

reliability ranges from $50 \%$ to $90 \%$ of all lobectomy cases depending on program maturity or surgeon preference for central, postinduction, or other complex tumors. Unfortunately, the Premier database does not have tumor data that could help control for these factors, which also influence pain.

Even with these data, dissecting approach from the surgical system is problematic, as demonstrated by inadequate nodal upstaging once attributed to VATS. This signal was likely biased by databases not capturing tumor centrality. ${ }^{6}$ The quality of lymph node dissection relates to surgeon and institutional normative practices, not to the approach per se. $^{7-9}$

Early adopters likely seek best practices when reengineering their techniques. Surgeons practicing the newer discipline of robotic lobectomy either already had VATS experience in avoiding pain or transitioned directly from an open approach. The latter group likely started with easier cases and mentoring from high-volume centers using optimized pain protocols. Hospital systems with resources to support robotic programs likely have other teams, such as enhanced recovery after surgery, that would favorably influence postthoracotomy care. The fact that the robotic patients in this study were more likely to receive early parenteral nonopioid interventions suggests these influences. 
Arguably, our taxonomy for minimally invasive surgery is no longer sufficient to tease out the complex mechanisms that cause postoperative pain. When a new system becomes popular, will we be comparing a Robot X lobectomy to "Intuitive lobectomy" to uniport to 2-port VATS, etc, to open? It is time to upgrade our databases with port placement details and other variables that relate mechanistically to postoperative pain. That way, all thoracic surgeons can benefit from the knowledge no matter what operative system works best for them.

\section{References}

1. Rajaram R, Rice DC, Li Y, Bruera E, Liu E, Song C, et al. Postoperative opioid use after lobectomy for primary lung cancer: a propensity-matched analysis of premier hospital data. J Thorac Cardiovasc Surg. 2021;162:259-68.e4.

2. Gross SD. System of Surgery. Vol 1. Philadelphia: Blanchard \& Lea; 1859.

3. Heister L. A General System of Surgery in Three Parts: Containing the Doctrine and Management. London: W. Innys; 1750.
4. Khoshbin E, Al-Jilaihawi AN, Scott NB, Prakash D, Kirk AJ. An audit of pain control pathways following video-assisted thoracoscopic surgery. Innovations (Phila). 2011;6:248-52

5. Oh DS, Tisol WB, Cesnik L, Crosby A, Cerfolio RJ. Port strategies for robotassisted lobectomy by high-volume thoracic surgeons: a nationwide survey. Innovations (Phila). 2019;14:545-52.

6. Decaluwé H, Horsleben Petersen R, Brunelli A, Pompili C, Agathe SeguinGivelet A, Gust L, et al. Multicentric evaluation of the impact of central tumour location when comparing rates of N1 upstaging in patients undergoing videoassisted and open surgery for clinical stage I non-small-cell lung cancer. Eur J Cardiothorac Surg. 2018;53:359-65.

7. Lee PC, Kamel M, Nasar A, Ghaly G, Port JL, Paul S, et al. Lobectomy for nonsmall cell lung cancer by video-assisted thoracic surgery: effects of cumulative institutional experience on adequacy of lymphadenectomy. Ann Thorac Surg. 2016;101:1116-22.

8. Krantz SB, Lutfi W, Kuchta K, Wang CH, Kim KW, Howington JA. Improved lymph node staging in early-stage lung cancer in the National Cancer Database. Ann Thorac Surg. 2017;104:1805-14.

9. Wolf A, Liu B, Leoncini E, Nicastri D, Lee DS, Taioli E, et al. Outcomes for thoracoscopy versus thoracotomy not just technique-dependent: a study of 9,787 patients. Ann Thorac Surg. 2018;105:886-91.

\section{Commentary: Can we embrace our bias and move beyond it?}

\section{Scott I. Reznik, MD}

One goal of any study is to eliminate bias. Responsible authors and editors watch constantly for sources of bias and use randomization or propensity matching to minimize bias. In this issue of the Journal, Rajaram and colleagues ${ }^{2}$ present an analysis of opioid use after robotic lobectomy (RL) compared with video-assisted thoracic surgery (VATS) and open lobectomy and report that fewer opioids are used in RL. The authors disclose their affiliations and the funding source. This may raise suspicion in the reader, since Intuitive Surgical (Sunnyvale, Calif) employs some of the authors, funded the study, and stands to benefit from results favoring robotic surgery. The authors have gone to great lengths to detail their efforts to eliminate

From the Department of Cardiovascular and Thoracic Surgery, UT Southwestern Medical Center, Dallas, Tex.

Disclosures: The author reported no conflicts of interest.

The Journal policy requires editors and reviewers to disclose conflicts of interest and to decline handling or reviewing manuscripts for which they may have a conflict of interest. The editors and reviewers of this article have no conflicts of interest.

Received for publication May 20, 2020; accepted for publication May 20, 2020; available ahead of print June 6, 2020.

Address for reprints: Scott I. Reznik, MD, Department of Cardiovascular and Thoracic Surgery, UT Southwestern Medical Center, 5323 Harry Hines Blvd, MC 8879 Dallas, TX 75390-8879 (E-mail: scott.reznik@utsouthwestern.edu).

J Thorac Cardiovasc Surg 2021;162:272-3

$0022-5223 / \$ 36.00$

Copyright (C) 2020 by The American Association for Thoracic Surgery

https://doi.org/10.1016/j.jtcvs.2020.05.088

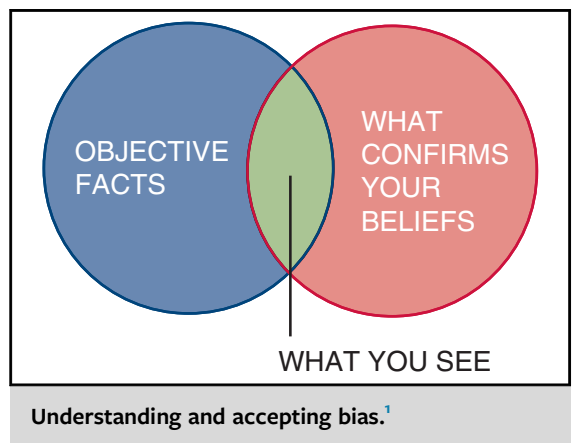

CENTRAL MESSAGE

Bias, recognized or not, may be present in almost every dataset.

Recognizing it and accounting

for it allows clinicians to accept

data and results when bias

cannot be eliminated.

bias, both real and perceived. The study protocol, available in the supplement, and statistical plan were created before institutional review board approval and data collection. The only deviation was a post-study sensitivity analysis requested during peer-review. The authors may have eliminated their bias, but can the reader but aside theirs? 